Chapter 8

\title{
Inflammatory Environmental, Oxidative Stress in Tumoral Progression
}

\author{
César Esquivel-Chirino, Jaime Esquivel-Soto, \\ José Antonio Morales-González, \\ Delina Montes Sánchez, Jose Luis Ventura-Gallegos, \\ Luis Enrique Hernández-Mora and \\ Alejandro Zentella-Dehesa
}

Additional information is available at the end of the chapter

http://dx.doi.org/10.5772/51789

\section{Introduction}

The incidence and prevalence of cancer has been increasing in such as degree that it has become the second or third leading cause of death worldwide, depending on ethnicity or country in question and is consequently a major public health, cancer is a leading cause of death in many countries, accounting for 7.6 million deaths (around 13\% of all deaths) in 2008. Deaths from cancer worldwide are projected to continue rising, with an estimated 13.1 million deaths in 2030 . About $30 \%$ of cancer deaths are due to the five leading behavioral and dietary risks: high body mass index, low fruit and vegetable intake, lack of physical activity, tobacco use, alcohol use [1-8].

Cancer is a generic term for a large group of diseases that can affect any part of the body, cancer cells are significantly influenced by the surrounding stromal tissues for the initiation, proliferation, and distant colony formation. When a tumour successfully spreads to other parts of the body and grows, invading and destroying other healthy tissues, this process is referred to as metastasis, and the result is a serious condition that is very difficult to treat, because the progression to metastases is the leading cause of death associated to cancer. Metastatic cells in this process must interact with the endothelium in three stages of tumor progression. In recent years, the interaction between these cell populations has been seen as part of a complex microenvironment tumor-associated. Mantovani et al. have even postulated that this tumor microenvironment inflammatory plays an important role in tumorigene- 
sis and tumor progression [18]. Tumor and normal surrounding cells such as endothelial cells, soluble factors derived from this two cell populations and extracellular matrix [9-12], compose the tumor microenvironment.

There are other factors in the development of cancer such as genetic, environmental, as well as the role of oxidative stress and free radicals in response to damage caused by chemicals, radiation, cellular aging, ischemic lesions and cells immune system [13]. Substances such as antioxidants can protect cells against free radical damage. The damage caused by free radicals can lead to cancer. Antioxidants interact with free radicals to stabilize them so that, being able to avoid some of the damage that free radicals can cause. It is important to analyze the role of antioxidants as an alternative that contributes to cancer treatment and to promote their use and consumption in cancer prevention

\section{Tumoral progression}

Tumors often become more aggressive in their behavior in more aggressive and their characteristics, although the time course may be quite variable, this phenomenon has been termed tumor progression by Foulds [15].

In the early stages of the tumor progression, there is a detachment of cancer cells from the primary tumor, followed by tumor cell adhesion to endothelial cells of venules in the target organs. After the extravasations occurs extracellular matrix invasion by tumor cells, these cells of primary lesions enter the lymphatics or the bloodstream depending on their anatomical location. In the circulation, many tumor cells are eradicated by physical forces exerted on them to pass through the microvasculature of secondary organs, and immunological mechanisms of action of host defense. Furthermore, once inside the target tissue tumor cells must find favorable conditions for survival and proliferation [16-18]. The biological characteristic that define tumor progression have been extensively described, although the underlying mechanisms are still not completely defined, however there are two theories have been proposed to explain how tumor cells invade secondary sites where metastasis occurs are the following [18-20]. The first is similar to the inflammatory process by cell adhesion and migration, while the second involves the aggregation of circulating tumor cells, and that these cells blocked blood vessels. In this theory in which a cell stably adhered frequently starts a homotypic aggregation, capturing other circulating tumor cells, followed by the formation of multicellular aggregates, these aggregates once grow and emerge from the primary tumor site, is carried out which triggers tumor progression in metastasis, where it requires a coordinated interaction of tumor cells and vascular endothelial cells, play a critical role in most of the events that characterize tumor progression and metastasis [21-23], so it is important to mention the general aspects of endothelial biology:

\subsection{Endothelial Biology}

The endothelium is the thin layer of cells that lines the interior surface of blood vessels and lymphatic vessels, forming an interface between circulating blood or lymph in the lumen 
and the rest of the vessel wall. The cells that form the endothelium are called endothelial cells, these cells have very distinct and unique functions that are paramount to vascular biology. These functions include fluid filtration, formation of new blood vessels in the angiogenesis, neutrophil recruitment. The endothelium acts as a semi-selective barrier between the vessel lumen and surrounding tissue, controlling the passage of materials and the transit of white blood cells, hormones into and out of the bloodstream. Excessive or prolonged increases in permeability of the endothelial monolayer, as in cases of chronic inflammation, may lead to tissue edema. It is also important in controlling blood pressure, blood coagulation, vascular tone, degradation of lipoproteins an in the secretion of growth factors and cytokines [24-25]. In recent decades, it has become clear that the endothelium of venules and smaller capillaries, and lymphatic vessels play a central role in the process of tumor growth, dissemination of metastatic cells, which is accompanied by the development of a characteristic tumor vasculature and tumors formed by endothelial cells [26].

There are two phenotypes endothelial (constitutive and activated);

\subsubsection{The constitutive phenotype of endothelial cells}

Quiescent, resting endothelial cells in the adult form a highly heterogeneous cell population that varies not only in different organs but also in different vessel calibers within an organ. Endothelium in the normal adult male, although being metabolically active, considered quiescent because the turnover of these cells is very low and this called: constitutive phenotype Fig (1).

In this condition, the apical membrane of endothelial cells exhibits a very low amount of intercellular adhesion molecules, so that no adhesion of cellular blood components to the vessel walls [27].

\subsubsection{The activated phenotype of endothelial cells}

Endothelial cell activation is associated with a number of distinct phenotype changes that, much like differentiation processes of the constitutive phenotype of endothelial cells, serve their need to adapt to functional requirements. [28] The cytokine-induced phenotype of endothelial cells during inflammation has been characterized most extensively in the last few years. When endothelial cells are activated by these cytokines are functional disorders involving immediate responses, for example, some pathological conditions such as sepsis, are associated with endothelial conversion to a phenotype activated [29-30]. The activated phenotype characterized by activation of constitutive nitric oxide synthase (NOS), also accompanied by changes such as increased expression of cell adhesion molecules (CAMs) and Eselectin (CD62E), ICAM-1(CD54), VCAM-1(CD106), P-selectin (CD62P) Fig (1). These changes allow the endothelium to participate in pathological conditions including inflammation, coagulation, cell proliferation, metastasis, tumor angiogenesis. All these cellular interactions are regulated by temporal and spatial presentation of various cell adhesion molecules and chemotactical molecules displaying appropriate specificity and affinity for 
proper development and functioning of the organism [31-32]. Has been postulated that this phenotype or variants of it, are involved in the processes of metastasis [33].

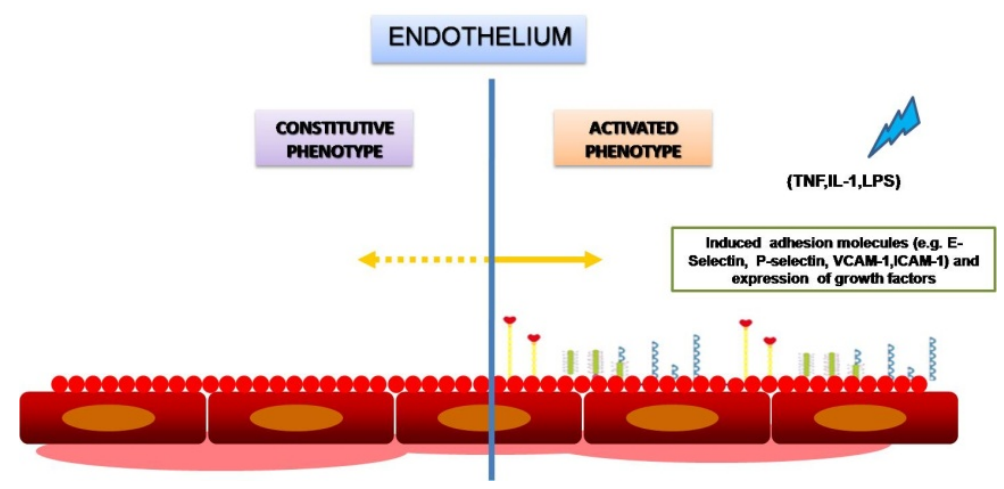

Figure 1. There are two phenotypes endothelial constitutive and activated.

\subsection{Metastasis}

Metastasis is the result of cancer cell adaptation to a tissue microenvironment at a distance from the primary tumor, is a complex process involving multiple steps: first, when cancer cells break away from the primary tumor, they invade the host stroma, intravasate into lymphatic or blood vessels, spread to the capillary bed of distant organs, where they invade into new surrounding tissues and proliferate to form secondary tumors [34-35]. When cancer is detected at an early stage, before it has spread, it can often be treated successfully by conventional cancer therapies such as surgery, chemotherapy, local irradiation, metastatic diffusion of cancer cells remains the most important clinical problem, because when cancer is detected after known to have metastasized, treatment are much less successful [36]. The metastatic capacity of tumor cells correlates with their ability to exit from the blood circulation, to colonize distant organs, and to grow in distant organs. Metastasis is a complex process that includes local infiltration of tumor cells into the adjacent tissue, transendothelial migration of cancer cells into vessels known as intravasation, survival in the circulatory system, extravasation and subsequent proliferation in competent organs leading to colonization [36-38]. Initially, tumor cell aggregates detachment from the primary tumor, next the cells actively infiltrate the surrounding stroma and enter into the circulatory system, traveling to distinct sites to establish the secondary tumor growth. In the bloodstream, a very small number of tumor cells survive to reach the target organ, indicating that metastasis formation must be regarded as a very ineffective event. Millions of carcinoma cells enter into the circulatory system, but the majority of them die during transportation, and only $1-5 \%$ of viable cells are successful in formation of secondary deposits in distinct sites [37-40]. Fig (2). 


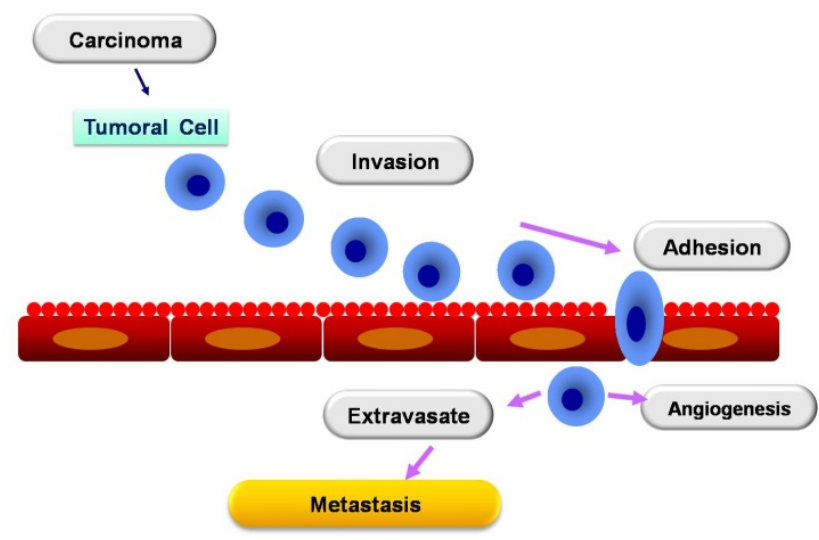

Figure 2. Steps in the Metastasis process.

Metastasis is facilitated by cell-cell interactions between tumor cells and the endothelium in distant tissues and determines the spread. Metastatic cells must act with the endothelium in three different stages of tumor progression: initially during the formation of blood vessels that enable tumor growth (vascularization), during the migration process that allows the passage from tissue into the bloodstream (intravasation), and finally during the process allowing extravasation into the target tissue [41-43]. Metastatic cancer cells require properties that allow them not only to adapt to a foreign microenvironment but also to subvert it in a way that is conducive to their continued proliferation and survival [36-38]. In addition, direct tumor cell interactions with platelets, fibroblasts and monocytes/macrophages, polymorphonuclear cells, soluble components, cytokines, chemokines, proteins of the extracellular matrix, growth factors, and other molecules secreted by host cells, significantly contribute to cancer cell adhesion, extravasation, and the establishment of metastatic lesions [44-47].

\subsection{Cellular interactions in the inflammatory reaction and spread tumor}

In the early stages of inflammation, neutrophils are cells that migrate to the site of inflammation under the influence of growth factors, cytokines and chemokines, which are produced by macrophages and mast cells residing in the tissue [48]. The process of cell extravasation from the bloodstream can be divided into four stages:

1. bearing

2. activation by stimulation chemoattractant

3. adherence

4. transendothelial migration.

If the inflammatory response is not regulated, the cellular response will become chronic and will be dominated by lymphocytes, plasma cells, macrophages metastasis, which is favored by the microenvironment of the organ target. The installation of tumor cells in blood vessels 
of the organ target to invade, is related to phenotypic changes in the endothelium allowing vascular extravasation of blood circulation of leukocytes in the inflammatory reaction and, as hypothesized current of tumor cells with metastatic capacity. The phenomenon of extravasation in response to a tumor cell interaction cell endothelial or not allowing the passage of cells whether there are appropriate conditions for the invasion with varied morphology [53-55].

Within the process of inflammation, a phenomenon is well-studied cell migration, which is the entrance of polymorphonuclear neutrophils and the vascular system. This involves a sequential mechanism of recognition, contact formation, and migration mediated by adhesion molecules such as (ICAM-1, VCAM-1, E and P Selectins, Integrins) it has been demonstrated that some of these adhesion molecules, such as E-selectin are not only involved in inflammation, but also in tumor metastasis and play a significant role in cancer progression and metastasis, in some cases of colon cancer. [56-58]. The expression of ICAM-1 has also proven to be a marker associated with an invasive phenotype [59].

Hanahanan et al. suggest that diversity of cancer cell genotypes is a manifestation of six basics alterations in cell physiology that together indicate development of malignant growth Fig (3), these alterations are shared in common by most all types of tumors [19]. In recent years, it has been demonstrated that metastatic dissemination can be influenced by inflammatory-reparative processes [46]. The interaction between these cell populations has been seen as part of a complex inflammatory microenvironment tumor-associated. Mantovani et al. have even postulated that this tumor microenvironment inflammatory plays an important role in tumorigenesis and tumor progression [60].

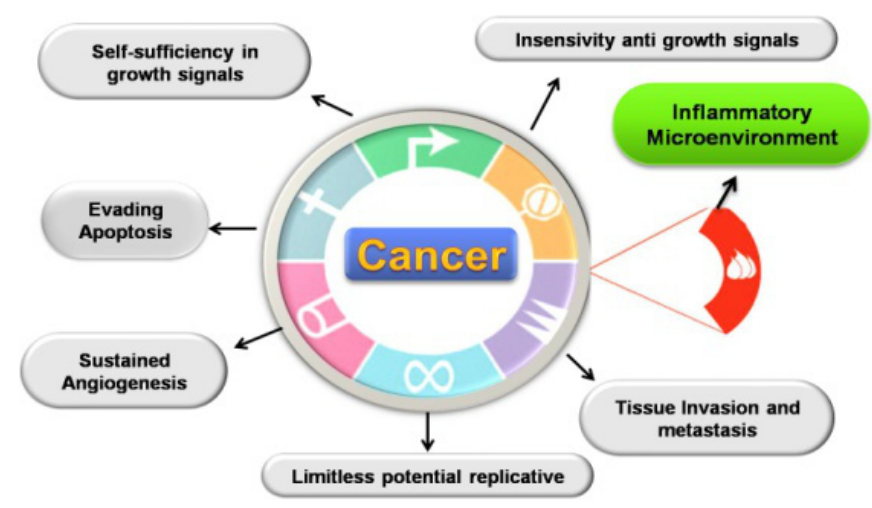

Figure 3. Capabilities of cancer and inflammation [19,60].

\subsection{Inflammatory Microenvironment}

The tumor microenvironment is composed of stromal fibroblasts, myofibroblasts, myoepithelial cells, macrophages, endothelial cells, leucocytes, and extracellular matrix (ECM) and soluble factors derived from tumor cells. This inflammatory environment surrounding a tumor promotes the breaking of the basal membrane, a process required for the invasion and 
migration of metastatic cells [60]. Tumor cells are also capable of produce cytokines and chemokines that facilitate evasion of the system immune and help to establishment and development of metastasis (Fig. 4). The increase of tumor-associated macrophages (TAMs) is associated with poor prognosis through various mechanisms: a) release by macrophage IL-10 and prostaglandin E2 which suppress antitumor response, b) easy to release angiogenic factors as VEGF, EGF, endothelin-2 and plasminogen activator promote tumor growth, c) to facilitate cell invasion metastasis by releasing matrix metalloproteinases and induce TNF production and vasodilatation enzyme nitric oxide synthase [61-62].

\section{Inflammatory Microenvironmental}

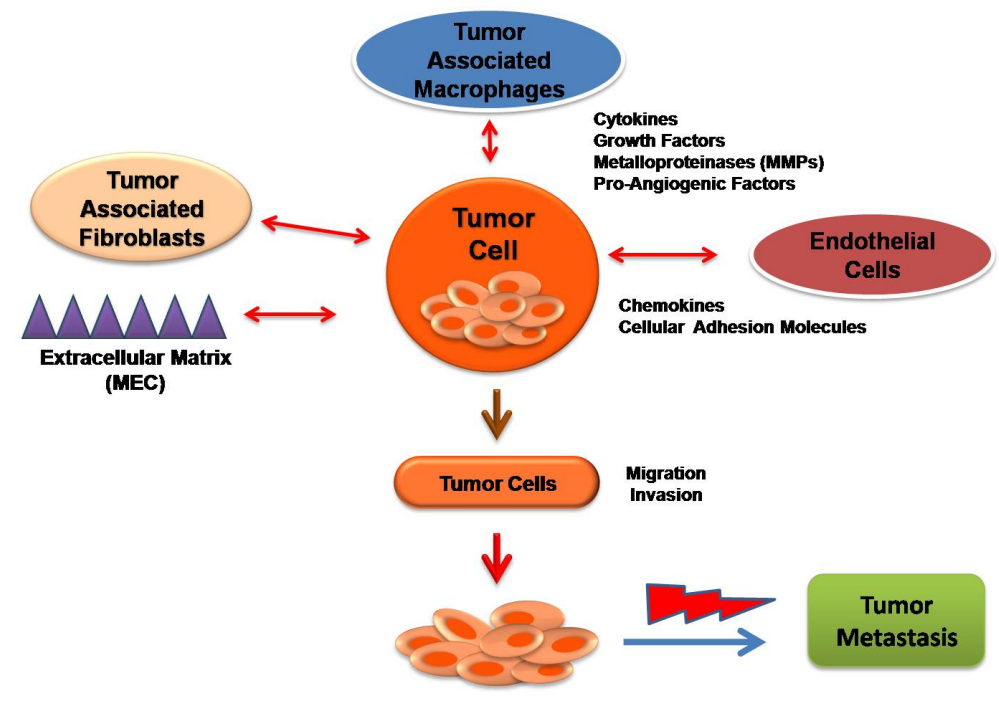

Figure 4. The tumor microenvironment and its role in promoting tumor growth

Cells grow within defined environmental sites and are subject to microenvironmental control. Outside of their sites, normal cells lack appropriate survival signals. During tumor development and progression, malignant cells escape the local tissue control and escape death. Diverse chemoattractant factors promote the recruitment and infiltration of these cells to the tumor microenvironment where they suppress the antitumor immunity or promote tumor angiogenesis and vasculogenesis.

TNF is expressed in low amounts by other cells such as fibroblasts, smooth muscle cells and tumor cells, his target cell are primary endothelial cells, inducing their activation by changing expression levels of some membrane proteins, primarily as adhesion molecules E-selectin, ICAM-1 and VCAM-1 whose expression and synthesis, are regulated by the transcription factor $\mathrm{kB}$ nuclear. Activated endothelial phenotype induced by TNF and characteristics of the inflammatory response, have served to comparing the endothelial pheno- 
type has been observed, is produced in response to contact with soluble tumor factors [63-64].

The nuclear factor kappa B (NF-kB) is a transcription factor paramount in regulation of inflammatory response genes Early involved in cell-cell interaction, communication intercellular recruitment or transmigration, amplification of signals pathogenic and acceleration of tumorigenesis [52-53,65].

The study of tumor cells to modify their microenvironment has been a growing area of interest, which has identified the secretion of pro-inflammatory cytokines such as TNF, IL-6) and chemokines such as IL-8, it is interesting to note that these products are known modulators of endothelial function [53].

In recent years, it has been found that tumor cells secrete soluble factors, which modify the endothelial constitutive phenotype, and that exposure to these factors increase to a greater or less extent the capacity to adhere endothelial human tumor cells. It has been recognized that these soluble factors released by tumor cells or non-tumor cells surrounding the tumor play an important role in tumor progression [66].

Our group has shown that breast cancer cells, lymphomas, with high metastatic potentially induces a change in human endothelial cells (HUVECs) that is characterized by promoting a pro-adhesive endothelial phenotype, the expression of intercellular adhesion molecules (ICAM-1, VCAM-1 and E-Selectin) and the activation of NF-kB [66]. These studies include soluble factor leukemias (EUHE, Eusebia), cervical cancer (HeLa) and mammary gland cancer (MCF-7, ZR), oral cancer. In all cases, we have used primary cultures of human endothelial cells (HUVECs), which have generated an in vitro model to study the tumor microenvironment. This model is based on induction of a pro-adhesive endothelial phenotype that is associated with expression of adhesion molecules E-selectin, ICAM-1, VCAM dependent activation of NF-kB. These effects are considered essential in the process of adhesion and extravasation during the inflammatory reaction.

Moreover, we have analyzed the biochemical composition of the soluble factors derived from tumor cells. Proteins such as 27, cytokines, chemokines and growth factors associated with the inflammatory reaction; (IL-1 ra, IL-1 beta, IL-2, IL-4, IL-5, IL-6, IL-7, IL-9, IL-10, IL-12, IL-13, IL-15, IL-16, TNF, IFN-gamma, IP-10,RANTES), (IL-8, Eotaxina, MCP-1, MIP-1 beta, MIP-1 alpha), (G-CSF, GM-CSF, PDGF, FGF, VEGF). The molecules identified most significant expression in breast carcinomas, were (VEGF, GM-CSF, IL-1ra, IL6, IL8, IP10, RANTES), which play an important role in endothelial cell activation [52-53,65-66].

\section{VEGF:}

An unexpected finding was the abundant presence of the expression of vascular endothelial growth factor (VEGF) in breast carcinoma lines. Whereas in normal cells the expression of VEGF is dependent on a condition of hypoxia, surprisingly VEGF production by tumor cell lines and occurred at a concentration of oxygen partial indicating 20\% indicative of impaired VEGF expression. In cancer constitutive secretion of VEGF independent of a condition that favors hypoxic tumor angiogenesis and growth in a clinical setting and is a marker of poor prognosis 
[67]. In cancer, constitutive secretion of VEGF-independent hypoxic condition favors tumor angiogenesis and growth in a clinical setting and is a marker of poor prognosis [68].

\section{GM-CSF}

The involvement of GM-CSF (Granulocyte Macrophage Colony Stimulating Factor) in cancer is complex, since it seems to require the presence of other cytokines such as IL-4 and IL-6, and there are reports antagonistic to their involvement in tumorigenesis. For example, expression of GM-CSF and interleukin-12, inhibits the immune response and being expressed on B16 murine melanoma cells, decreasing completely tumorigenic capacity of syngenic mice $\mathrm{C} 57 \mathrm{~B} / 6$. In contrast to the line described breast epithelial MCF7-A benign growth factors secreted able to induce expression of IL-6 and GM-CSF in the tumor line R2T1AS breast cancer that is associated with a higher rate of growth and a higher tumorigenic capacity in vivo. Moreover, continued exposure of GM-CSF plus IL-4 of mesenchymal cells from human bone marrow, resulted in an increase in the morphological transformation and increased rate of growth both in vitro and in vitro mesenchymal cells, indicating the induction of a transformed phenotype. Whereas previous reports indicate that the involvement of GM-CSF requires other cytokines [69-70].

\section{IL-1ra}

It is reported that the soluble form of IL-1 receptor could serve as a functional antagonist of IL-1, the presence of this protein is controversial, since IL- 1 by trapping an anti-inflammatory effect would reduce the adhesion of cells added by tumor cells and their extravasation, given this scenario could be proposed that tumor cells not only produce pro-inflammatory factors through the release of soluble factors tumor, but it can regulate the inflammatory process [71].

IL-6

The IL-6 is recognized as a classic inducer of the states of chronic inflammation and that promote the activation of vascular endothelium. It has also been reported that IL-6 is produced by tumors that develop metastases to the liver, as the case of colon and mammary gland cancer. The activity of this cytokine in the soluble factors tumor could be further enhanced by the presence of other co-factors secreted by cells [72-73].

\section{IL-8}

Expression of IL-8 in colorectal cancer favors an increase in tumorigenesis and metastasis, this increase is due to IL- 8 is associated with expression of MMP-2 and MMP-9, the activity of these metalloproteases have been identified in physiological tissue remodeling processes like normal healing, but also participate in tissue remodeling associated with pathological processes including invasion [74]. Also in melanomas have been identified as IL-8 acts as an angiogenic factor and also promotes mitosis, therefore the IL- 8 has been postulated as a potential therapeutic target. In particular, we have sought to interfere with IL- 8 with the purpose of reducing tumor growth, an alternative that has developed is the use of an anti-IL- 8 in nude mice with liver cancer. The results show that administration of neutralizing anti-IL8 significantly decreased tumor growth, even more interestingly this decrease is associated 
with decreased expression of MMP-2 and MMP-9. Something similar is observed using the same experimental treatment of melanoma with a decrease in angiogenesis [75]. In our results, we found increases in IL-8 in the soluble factors soluble breast cancer [66]. This indicates that IL-8 could be used as a marker associated with tumor progression, regardless of tumor type. In patients where they identified the production of IL-8, using neutralizing antibodies that interfere with their signaling could serve as a therapeutic alternative.

IP10

IP10 is a protein induced by interferon, has been reported that this protein inhibits proliferation and metastatic tumors, that expression of IP10 in patients with stages II and III colorectal cancer correlate with the development of metastasis and a poor prognosis. The detection of IP10 could be used as a prognostic marker in stage II and III colorectal cancer patients. This has led to propose that IP10 might be used as a prognostic marker in stage II and III colorectal cancer patients [76-77].

\section{RANTES}

Regulated upon activation normal T-cell expressed, and secreted is a chemokine that belongs to the CC class, which distinguishes it from IL8, which belongs to the CXC class. RANTES expression in tumor cells has been associated with tumorigenesis and is consistent with our finding of RANTES the products secreted by breast carcinomas. The presence of RANTES in the tumor microenvironment may be chemoattractant to tumor cells. From this point of view is interesting that the tumor-associated endothelial cells, when stimulated with MIF1-alpha can release RANTES [78]. Study that evaluated the expression of RANTES and its receptor CCR5 in 60 patients with metastatic gastric cancer, were identified elevated expression levels, where it is concluded that RANTES and its receptor may contribute to gastric cancer metastasis by promoting responses TH1 and TH2. By comparing a variety of biological markers in a group of biopsies of mammary gland cancer, RANTES was the only marker present in all biopsies [79].

The reported findings strengthen the idea that soluble factors of tumor microenvironment may be relevant in the final stages of the metastatic spread and that these effects may be mediated by cytokines, chemokines, and growth factors present in the soluble factors secreted by tumor cell lines. These elements found in high concentrations are known to be capable of inducing the activated phenotype of endothelial cells to a variety of physiological and pathological cellular responses.

\section{Oxidative stress and free radicals: role in cancer development}

During the inflammatory process macrophages and endothelial cells, generate a large amount of growth factors, cytokines and reactive oxygen species (ROS) and nitrogen (RNS) that can cause DNA damage. If macrophages and remain on the endothelium may allow the tissue damage continues chronic inflammation predisposes to malignancy $[56,80]$. 


\subsection{Introduction}

In different pathological process the cell injury is induced by free radicals, is an important mechanism of cell damage in many pathological conditions, such as chemical and radiation injury, ischemia injury, cellular aging and some immune system cells such as the phagocytes [82-84]. The free radicals are an example of instability in a biological system; namely, are chemical substances that they have an unpaired electron in its final orbit, this causes that their energy to be unstable and for they become stable they need for molecules which are adjacent to it either organic or inorganic for example: lipids, proteins, carbohydrates and nucleic acids essentials compounds in the cells mainly in the membrane and the core.

These types of chemical species may be either.

1. Oxygen derived (reactive oxygen species ROS)

2. Nitrogen derived (reactive nitrogen species RNS)

Reactive oxygen species (ROS) and reactive nitrogen (RNS) (Table 1) are created in the some cells such as the hepatocytes and in different normal physiologic processes, including, oxidative respiration, growth, regeneration, apoptosis and microbial killing by phagocytes [83]. The generation of this species chemical types, is normal in a normal cells; however, when these start to produce in excess and the antioxidant system is deficient, oxidative stress occurs. This causes damage cells: hepatocytes, kupffer cells and endothelial cells, through induction of inflammation, ischemia, fibrosis, apoptosis, necrosis or other atypical transformation in the cell structure and function.

\begin{tabular}{lll}
\hline Type of radical & Activating enzyme & Physiological process \\
\hline Nitrogen derived & Nitric oxide synthase & Smooth muscle (control or vascular tone) and other \\
(nitric oxide) & NADPH oxidase & cGMP- depended functions. (glycogenolysis, apoptosis, \\
Oxygen derived & conductance regulator ion channels, vasodilatation \\
(ROS) & and increased blood flow) \\
& Oxidation-reduction reactions within the cell, muscle \\
& relaxation, control of erythropoietin production, signal \\
& transduction from various receptors, enhancement of \\
& immunological functions and oxidative
\end{tabular}

Table 1. Important physiological process involved with the free radicals.ROS, Reactive oxygen species.

\subsection{Reactive oxygen species}

Reactive oxygen species are produced in normal condition them in a living cell during cellular respiration, energy production and various events of growth and cell death, these are degrade by the defensive systems. Therefore, the cells self-regulate their production and degradation of ROS is found transiently in the cell without causing any damage to the cellular level, and for that reason the cell maintains an equilibrium constant but as this production increases oxidative stress is generated, this relates whit different pathological process 
such as damage in the cell structure and function, degenerative process and cancer, also influence within the inflammation and the immune response, as these are generated by macrophages and neutrophils as mediators for the destruction of pathogens and dead tissue. The generation of free radicals can be made by different pathways [85-86]. (Table 2)

\begin{tabular}{|c|c|}
\hline Redox reactions in metabolic processes. & $\begin{array}{l}\text { During cellular respiration } \mathrm{O}_{2} \text { is reduced by four } \\
\text { electrons to the transport of } \mathrm{H}_{2} \text { for generating two } \\
\text { molecules of water through an oxidative enzyme } \\
\text { which results is the formation of superoxide anion } \\
\text { (electron), hydrogen peroxide (two electron ) and } \\
\text { hydroxyl ions (three electrons). }\end{array}$ \\
\hline Absorption of radiant energy & $\begin{array}{l}\text { Electromagnetic radiation ( } x \text { rays), gamma rays, } \\
\text { infrared, UV, microwave etc. These to hydrolyze the } \\
\text { water and generate hydroxyl ions and hydrogen }\end{array}$ \\
\hline Inflammation and immune response. & $\begin{array}{l}\text { This response induced by activated leukocytes, that } \\
\text { is caused by a protein complex located at the } \\
\text { plasma membrane that employs NADPH oxidase } \\
\text { and some intracellular oxidase and this generated a } \\
\text { superoxide anion. }\end{array}$ \\
\hline Metabolism of drugs & $\begin{array}{l}\text { Most chemicals do not show biological activity in its } \\
\text { native form these have to become toxic reactive } \\
\text { metabolites to act on their target molecules. This is } \\
\text { made by oxidases. These metabolites induce the } \\
\text { formation of free radicals, such as acetaminophen. }\end{array}$ \\
\hline Metals & $\begin{array}{l}\text { The transmission metals donate or accept electrons } \\
\text { intracellular free during the reactions thus causing } \\
\text { the formation of free radicals }\end{array}$ \\
\hline Nitric oxide & $\begin{array}{l}\text { Nitric oxide is generated by different cells are an } \\
\text { example of this are the endothelial cells giving rise } \\
\text { to reactive species of nitrogen, such as the } \\
\text { peroxynitrite anion (ONOO-) also } \mathrm{NO}_{2} \text { and } \mathrm{NO}_{3} \text {. }\end{array}$ \\
\hline
\end{tabular}

Table 2. The generation of free radicals can be made by different pathways.

\subsection{Free radical and carcinogenesis}

Free radicals are atoms or groups of atoms that in their atomic structure present one or more unpaired electrons in the outer orbit. These free radicals steal electrons from other molecules in effort to heal themselves, ultimately creating new free radicals in the process by stealing electrons. Free radicals are formed from a number of causes such as cigarette smoke, pollution, exposure to sunlight all cause the formation of free radicals. Other factors include normal daily processes like food digestion and breathing. 
When increased production of reactive species and have a deficiency in the antioxidant system they cause significant neoplastic changes. In some diseases, such as Bloom syndrome develops lymphomas, leukemias and carcinomas, in anemia are implicated the production of these and alterations of antioxidant defense mechanisms at the systemic level [82-83]. Some epidemiological information indicates that tumor incidence is lower in populations where the diet is rich in antioxidants like fruits and vegetables [84].

Tumor cells have a high activity of free radical formation in contrast to healthy cells. It is known that tumor cells not only produce oxygen peroxide $\left(\mathrm{H}_{2} \mathrm{O}_{2}\right)$ but also decreases the production of antioxidants such as glutathione peroxidase and SOD.

Some pathways by which cancer cells have high amounts of ROS is for multiple factors:

Increasing the metabolic activity of a neoplastic cell, lead to the increased energy requirements (ATP) produced in the mitochondria this in order to enhance the growth and proliferation.

1. The progression of cancer, primarily because of the damage they cause in to the genetic material of a normal cell. Has been shown that the oxidation of guanine to 8-oxo-dG (oxidation product DNA) induces errors in their replication. By dependent of DNA polymerase that generates the nitrogenous base pairing, not complementary, to permit the establishment of hydrogen bonds with (A) adenine and (T) thymine [85].

2. Oncogenic transformation is conditioned by the presence of mutated genes or oncogenes that control essential cellular functions in which the redox state within or outside the cellular microenvironment is very important ROS are potential carcinogens because they facilitate mutagenesis, tumor growth, and metastasis and all those process that has been showed [86].

Cancer is a multifactorial disease where endogenous and exogenous factors are involved but the roles played by free radicals in this disease are very important because, these produce damage in de DNA structure and this produces an important negative effects [86].

\section{Antioxidants and Chemoprevention in cancer}

Antioxidants are substances that prevent damage to cells caused by free radicals, it can cause damage to DNA, leading to the possible development of cancer [87]. Antioxidants search for these free radicals and lend them an, this stabilizes the molecule, thus preventing damage to other cells. Antioxidants also turn free radicals into waste by products, and they eventually are eliminated from the body. The inability of our body to neutralize free radicals we are exposed daily forces us to rely on foods with antioxidant properties capable of neutralizing them [88]. 


\subsection{Flavonoids}

Flavonoids are found in numerous plants and vegetables, with a wide distribution through the plant kingdom. This class compounds numbers more than 4000 members and can be divided into five subcategories: flavones, monomeric flavanols, flavanones, flavonols and anthocyanidines. Are natural compounds chemically derivate from bezo-y-pirone (phenylchromone) or flavone. They are considered important constituents of the human diet. It has been reported that they exert multiple biological effects due to their antioxidant and free radical-scavenging abilities [89].

Flavanoids possess a lot of pharmacological and therapeutically properties; antioxidant, antitumor, antiangiogenic (vascular protective), anti-inflammatory, antiallergic, antihepatotoxic, anticancerigenic, antimutagenic, anticancer effects, antiosteoporotic, and antiviral properties [90]. Many studies emphasize take adequate diets that are active allies against cancer. These diets are based on enzymes and antioxidant substances in certain foods that are rich in components that collect above [91].

They also have the ability to repair previous damage to cells, examples of antioxidants include (beta-carotene, lycopene, vitamins C, E, and A), and other substances. Nutrients such as; green tea, flavonoids, vitamins $\mathrm{C}, \mathrm{E}$, and Beta-carotene in the carcinogen process, has been showed that have function in the elimination of carcinogenic factors, inhibition of precarcinogens and reparation of DNA damage. The mechanisms are diverse and range from inhibition to an active reaction of the immune system in general. This has caused the use of multiple antioxidant micronutrients as preventive agents [90]. Several experimental data have demonstrated the antiproliferative and anti-carcinogenic and the role of chemopreventive agent of flavonoids [91-92].

Currently investigations are performed to determine the mechanisms by which act flavonoids, because it has been observed that their effects are greater at high doses, which gives them inducing side effects, so it is important to moderate their consumption by a balanced diet.

\section{Conclusions}

It is important to analyze the role of tumor-associated inflammatory microenvironment and has been identified that plays an important role in tumor progression. This microenvironment is composed of molecules that play an important role in inflammatory processes and chronic, and favor the invasion and metastasis process that triggers the death of many people with any cancer.

The installation of tumor cells in blood vessels of the target organ to invade, is related to phenotypic changes in the endothelium allowing vascular extravasation of blood circulation of leukocytes in the inflammatory reaction and, as hypothesized current of tumor cells with metastatic capacity. The phenomenon of extravasation in response to a cell interactions be- 
tween tumor cells and endothelial cells or not allowing the passage of cells whether there are appropriate conditions for the invasion.

Understanding the molecular basis of these interactions between metastatic cells and endothelial cells, will enable us to design strategies to interfere with this inter-cellular communication. It is important to recognize the tumor-associated inflammatory microenvironment and what is the contribution to tumor progression. The importance of these factors on endothelial activation being evaluated by reconstituting the mixture with cytokines, chemokines and growth factors recombinant depleted mixtures of tumor soluble factors of each of these proteins by specific monoclonal antibodies.

Is important mention that during the inflammatory process macrophages, fibroblasts and endothelial cells generate a large amount of growth factors, cytokines, chemokines and reactive oxygen species (ROS) and nitrogen (RNS) that can cause DNA damage. These process allow the tissue damage continues chronic inflammation predisposes to malignancy. Therefore, it is important to note that people with chronic degenerative diseases, which clearly show chronic inflammatory processes, they may promote or contribute to present or develop a tumor lesion.

The use of antioxidants consumed in a balanced diet can be used as an element in the diet that can become a preventive or contributing to diminish the appearance of a tumor lesion.

\section{Author details}

César Esquivel-Chirino 1,4,5,6*, Jaime Esquivel-Soto ${ }^{1,6}$, José Antonio Morales-González², Delina Montes Sánchez ${ }^{3,4,5}$, Jose Luis Ventura-Gallegos ${ }^{4,5}$, Luis Enrique Hernández-Mora ${ }^{1}$ and Alejandro Zentella-Dehesa ${ }^{4,5}$

*Address all correspondence to: cesquivelch@gmail.com

1 Facultad de Odontología, Universidad Nacional Autónoma de México, México

2 Instituto de Ciencias de la Salud, Universidad Autónoma del Estado de Hidalgo (UAEH), México

3 Programa de Genómica Funcional de Procariotes, Centro de Ciencias Genómicas, Universidad Nacional Autónoma de México, Campus Morelos, México

4 Departamento de Medicina y Toxicología Ambiental, Instituto de Investigaciones Biomédicas. Universidad Nacional Autónoma de México, México

5 Departamento de Bioquímica. (INNCMSZ) Instituto Nacional de Ciencias Médicas y Nutrición “Salvador Zubirán” México D.F., México

6 Facultad de Odontología, Universidad Intercontinental, México 


\section{References}

[1] Ferlay, J., Bray, P., Pisani, P., \& Parkin, D. M. (2004). GLOBOCAN 2002: Cancer incidence, mortality and prevalence worldwide. IARC Cancer Base Version 2.0. Lyon: IARC Press [5].

[2] World Health Organization. (2008). World cancer report 2008. Lyon (France): IARC.

[3] World Health Organization. (2007). Ten statistical highlights in global public health. World health statistics 2007. Geneva: WHO.

[4] Jemal, A., Ward, E., \& Thun, M. (2005). Cancer statistics. In: DeVita VJ, Hellman S, Rosenberg $S$, editors. Cancer principles and practice of oncology. 7 th ed. Baltimore (MD): Lipppincott Williams \& Wilkins, 226-240.

[5] International Agency for Cancer Research (IARC). (2012). CANCER Mondial. available from: http://www-dep.iarc.fr/Accessed on June 22

[6] Jemal, A., Bray, F., Center, M. M., Ferlay, J., Ward, E., \& Forman, D. (2011). Global cancer statistics. CA Cancer J Clin, 61, 69-90.

[7] Kanavos, P. (2006). The rising burden of cancer in the developing world. Ann Oncol, $17(8), 15-23$.

[8] Kolonel, L., Wilkens, L., Schottenfeld, D., \& Fraumeni, J. F. Jr. (2006). Cancer epidemiology and prevention. 3rd ed Oxford: Oxford University Press, 189-201.

[9] Bacac, M., \& Stamenkovic, I. (2008). Metastatic cancer cell. Annu Rev Pathol, 3, 221-47.

[10] Jackson, S. P., \& Bartek, J. (2009). The DNA-damage response in human biology and disease. Nature, 461, 1071-1078.

[11] Pantel, K., Brakenhoff, R. H., \& Brandt, B. (2008). Detection, clinical relevance and specific biological properties of disseminating tumor cells. Nat Rev Cancer, 8, 329-340.

[12] Hanahan, D., \& Weinberg, R. (2000). The hallmarks of cancer. Cell, 100, 57-70.

[13] Oldham Hikman, Elizabeth. (2004). Intrinsic oxidative stress in cancer cells a biological basis for therapeutic selectivity" Cancer". Cancer chemother pharmacol, 53, 209-19.

[14] Steinmetz, K. A., \& Potter, J. D. (1991). Vegetables, fruit, and cancer. I. Epidemiology. Cancer Causes Control., 2, 325-357.

[15] Foulds, L. (1954). The experimental study of tumor progression: a review. Cancer Res, $14,327-339$.

[16] Ann, F., Chambers, Alan. C., \& Groom, Iand. Mc Donald. (2002). Dissemination and growth cancer cells in metastatic sites. Nature Reviews, 2.

[17] Alby, L., \& Auerbach, R. (1984). Differential adhesion of tumor cells to capillary endothelial cells in vitro. Proc Natl Acad Sci USA, 81, 5739-43. 
[18] Balkwill, F., \& Mantovani, A. (2001). Inflammation and cancer: back to Virchow? Lancet, $357,539-45$.

[19] Hanahan, D., \& Weinberg, R. A. (2011). Hallmarks of cancer: the next generation. Cell., 44, 646-674.

[20] Chaffer, C.L, \& Weinberg, R. A. (2011). A perspective on cancer cell metastasis. Science. Mar 25; , 331(6024), 1559-64.

[21] Nicolson, G. L. (1993). Cancer progression and growth: relationship of paracrine and autocrine growth mechanisms to organ preference of metastasis. Exp Cell Res, 204, $171-80$.

[22] Kopfstein, L., \& Christofori, G. (2006). Metastasis: cell-autonomous mechanisms versus contributions by the tumor microenvironment. Cell Mol Life Sci., 63(4), 449-68.

[23] Calorini, Lido, \& Bianchini, Francesca. (2010). Environmental control of invasiveness and metastatic dissemination of tumor cells: the role of tumor cell-host cell interactions. Cell Communication and Signaling, 8, 24.

[24] Tang, D. G., \& Conti, C. J. (2004). Endothelial cell development, vasculogenesis, angiogenesis, and tumor neovascularization: an update. Semin Thromb Hemost., 30(1), 109-17.

[25] Aird, W. C. (2009). Cell Tissue Res. Molecular heterogeneity of tumor endothelium. Epub 2008 Aug 23., 335(1), 271-81.

[26] Risau, W. (1995). Differentiation of endothelium. FASEB J, 9, 926-33.

[27] Ribatti, Domenico, Nico, Beatrice, Vacca, Angelo, Roncali, Luisa, \& Dammacco, Franco. (2002). Journal of Hematotherapy \& Stem. Cell Research., 11(1), 81-90.

[28] Augustin, H. G., Kozian, D. H., \& Johnson, R. C. (1994). Differentiation of endothelial cells: Analysis of the constitutive and activated endothelial cell phenotypes. Bioessays, 16, 901-906.

[29] Geng, J. G. (2003). Interaction of vascular endothelial cells with leukocytes, platelets and cancer cells in inflammation, thrombosis and cancer growth and metastasis. Acta Pharmacol Sin., 24(12), 1297-300.

[30] Pober, J.S. (2002). Arthritis Res. 4(3), 109-16, Epub May 9. Endothelial activation: intracellular signaling pathways.

[31] Riscoe, D. M., Cotran, R. S., \& Pober, J. S. (1992). Effects of tumor necrosis factor, lipopolysaccharide, and IL- 4 on the expression of vascular cell adhesion molecule- 1 in vivo. Correlation with CD3+ T cell infiltration. J Immunol., 149, 2954-2960.

[32] Joan, M., \& Cook-Mills, Tracy. L. Deem. (2005). Active participation of endothelial cells in inflammation. J Leukoc Biol., 77(4), 487-495. 
[33] Wagner, M., Bjerkvig, R., Wiig, H., Melero-Martin, J. M., Lin, R. Z., Klagsbrun, M., \& Dudley, A. C. (2012). Inflamed tumor-associated adipose tissue is a depot for macrophages that stimulate tumor growth and angiogenesis. Angiogenesis. May 22.

[34] Bendas, G, \& Borsig, L. (2012). Cancer cell adhesion and metastasis: selectins, integrins, and the inhibitory potential of heparins. Int J Cell Biol, 676-731.

[35] Chambers, A. F., Groom, A. C., \& Mac Donald, I. C. (2002). Dissemination and growth of cancer cells in metastatic sites. Nature Reviews Cancer, 2(8), 563-572.

[36] Calorini, Lido, \& Bianchini, Francesca. (2010). Environmental control of invasiveness and metastatic dissemination of tumor cells: the role of tumor cell-host cell interactions. Cell Communication and Signaling, , 8, 24.

[37] Rubin, H. (2008). Contact interactions between cells that suppress neoplastic development: can they also explain metastatic dormancy? Adv Cancer Res, 100, 159-202.

[38] Aguirre-Ghiso, J. A. (2007). Models, mechanisms and clinical evidence for cancer dormancy. Nat Rev Cancer, 7, 834-846.

[39] Nicolson, G. L. (1993). Cancer progression and growth: relationship of paracrine and autocrine growth mechanisms to organ preference of metastasis. Exp Cell Res, 204, 171-80.

[40] Gasic, G. J. (1986). Role of plasma, platelets and endothelial cells in tumor metastasis. Cancer Metastasis Rev, 3, 99-116.

[41] De Visser, K. E., Korets, L. V., \& Coussens, L. M. (2005). De novo carcinogenesis promoted by chronic inflammation is B lymphocyte dependent. Cancer Cell, 7, 411-423.

[42] Di Carlo, E., Forni, G., Lollini, P., Colombo, M. P., Modesti, A., \& Musiani, P. (2001). The intriguing role of polymorphonuclear neutrophils in antitumor reactions. Blood, 97, 339-345.

[43] De Larco, J. E., Wuertz, B. R., \& Furcht, L. T. (2004). The potential role of neutrophils in promoting the metastatic phenotype of tumors releasing interleukin- 8 . Clin Cancer Res, 10, 4895-4900.

[44] Silzle, T., Randolph, G. J., Kreutz, M., \& Kunz-Schughart, L. A. (2004). The fibroblast: sentinel cell and local immune modulator in tumor tissue. Int J Cancer, 108, 173-180.

[45] Kalluri, R., \& Zeisberg, M. (2006). Fibroblasts in cancer. Nat Rev Cancer, 6, 392-401.

[46] Nicolson, G. L. (1993). Cancer progression and growth: relationship of paracrine and autocrine growth mechanisms to organ preference of metastasis. Exp Cell Res, 204, 171-80.

[47] Mehlen, P., \& Puisieux, A. (2006). Metastasis: a question of life or death Nat. Rev. Cancer, 6, 449-458.

[48] Fidler, I. J. (2005). Cancer biology is the foundation for therapy. Cancer Biol. Ther., 4, 1036-1039. 
[49] Almog, N. (2010). Molecular mechanisms underlying tumor dormancy. Cancer Lett, 294, 139-146.

[50] Klein, C. A. (2009). Parallel progression of primary tumours and metastases. Nat. Rev. Cancer, 9, 302-312.

[51] Lu, H., Ouyang, W., \& Huang, C. (2006). Inflammation, a key event in cancer development. Mol Cancer Res, 4, 221-233.

[52] Lopez-Bojorquez, L. N. (2004). Regulation of NF-kappaB transcription factor. A molecular mediator in inflammatory process. Rev Invest Clin., 56, 83-92.

[53] Lopez-Bojorquez, L. N., Arechavaleta-Velasco, F., Vadillo-Ortega, F., Montes-Sanchez, D., Ventura-Gallegos, J. L., \& Zentella-Dehesa, A. (2004). NF-kappaB translocation and endothelial cell activation is potentiated by macrophage released signals cosecreted with TNF-alpha and IL-1beta. Inflamm Res., 53, 567-575.

[54] Smid, M., Wang, Y., Zhang, Y., Sieuwerts, A. M., Yu, J., Klijn, J. G., Foekens, J. A., \& Martens, J. W. (2008). Subtypes of breast cancer show preferential site of relapse. Cancer Res, 68, 3108-3114.

[55] $\mathrm{Wu}, \mathrm{J}$. M., et al. (2008). Heterogeneity of breast cancer metastases: comparison of therapeutic target expression and promoter methylation between primary tumors and their multifocal metastases. Clin Cancer Res, 14, 1938-1946.

[56] O'Hanlon, D. M, Fitzsimons, H, Lynch, J, Tormey, S, Malone, C, \& Given, H. F. (2002). Soluble adhesion molecules (E-selectin, ICAM-1 and VCAM-1) in breast carcinoma. Eur J Cancer., 38, 2252-2257.

[57] Kim, I., Moon, S. O., Kim, S. H., Kim, H. J., Koh, Y. S., \& Koh, G. Y. (2001). Vascular endothelial growth factor expression of intercellular adhesion molecule 1 (ICAM-1), vascular cell adhesion molecule 1 (VCAM-1), and E-selectin through nuclear factorkappa B activation in endothelial cells. J Biol Chem., 276, 7614-7620.

[58] Lieder, A. M., Prior, T. G., Wood, K. J., \& Werner, J. A. (2005). The relevance of adhesion molecules in the classification of 72 squamous cell carcinoma of the head and neck. Anticancer Res, 25, 4141-4147.

[59] Okegawa, T, Li, Y, Pong, R. C., \& Hsieh, J. T. (2002). Cell adhesion proteins as tumor suppressors. J Urol., 167, 1836-1843.

[60] Colotta, F., Allavena, P., Sica, A., Garlanda, C., \& Mantovani, A. (2009). Cancer-related inflammation, the seventh hallmark of cancer: links to genetic instability. Carcinogenesis., 30, 1073-81.

[61] Kawaguchi, T. (2005). Cancer metastasis: characterization and identification of the behavior of metastatic tumor cells and the cell adhesion molecules, including carbohydrates. Curr Drug Targets Cardiovasc Haematol Disord, 39-64. 
[62] Thorne, R. F., Legg, J. W., \& Isacke, C. M. (2004). The role of the CD44 transmembrane and cytoplasmic domains in coordinating adhesive and signalling events. J Cell Sci., 117, 373-380.

[63] Li, A., Li, H., Jin, G., \& Xiu, R. (2003). A proteomic study on cell cycle progression of endothelium exposed to tumor conditioned medium and the possible role of cyclin D1/E. Clin Hemorheol Microcirc., 29, 383-390.

[64] Watts, M. E., Parkins, C. S., \& Chaplin, D. J. (2002). Influence of hypoxia and tumourconditioned medium on endothelial cell adhesion molecule expression in vitro. Anticancer Res., 22, 953-958.

[65] Estrada-Bernal, A., Mendoza-Milla, C., Ventura-Gallegos, J. L., Lopez-Bojorquez, L. N., Miranda-Peralta, E., Arechavaleta-Velasco, F., Vadillo-Ortega, F., Sanchez-Sanchez, L., \& Zentella-Dehesa, A. (2003). NF-kappaB dependent activation of human endothelial cells treated with soluble products derived from human lymphomas. Cancer Lett, 191, 239-48.

[66] Montes-Sanchez, D., Ventura, J. L., Mitre, I., Frias, S., Michan, L., Espejel-Nunez, A., Vadillo-Ortega, F., \& Zentella, A. (2009). Glycosylated VCAM-1 isoforms revealed in 2D western blots of HUVECs treated with tumoral soluble factors of breast cancer cells. BMC Chem Biol, 9, 7.

[67] Baldewijns , M. M., van Vlodrop, I. J., Vermeulen, P. B., Soetekouw, P. M., van Engeland, M., \& de Bruïne, A. P. (2010). VHL and HIF signalling in renal cell carcinogenesis. J Pathol., 221(2), 125-38.

[68] Kamada, H., Tsutsumi, Y., Kihira, T., Tsunoda, S., Yamamoto, Y., \& Mayumi, T. (2000). In vitro remodeling of tumor vascular endothelial cells using conditioned medium from various tumor cells and their sensitivity to TNF-alpha. Biochemical and Biophysical Research Communications, 268, 809-813.

[69] Edeline, J., Vigneau, C., Patard, J. J., \& Rioux-Leclercq, N. (2010). Signalling pathways in renal-cell carcinoma: from the molecular biology to the future therapy]. Bull Cancer, 97, 5-15.

[70] Kulbe, H., Chakravarty, P., Leinster, D. A., Charles, K. A., Kwong, J., Thompson, R. G., Gallagher, W. M., Galletta, L., Salako, M. A., Smyth, J. F., Hagemann, T., Brennan, D. J., Bowtell, D. D., \& Balkwill, F. R. (2011). A dynamic inflammatory cytokine network in the human ovarian cancer microenvironment. Cancer Res.

[71] Friberg, E., Orsini, N., Mantzoros, C. S., \& Wolk, A. (2007). Diabetes mellitus and risk of endometrial cancer: a meta-analysis. Diabetologia., 50(7), 1365-74.

[72] Mc Lean, M. H., Murray, G. I., Stewart, K. N., Norrie, G., Mayer, C., Hold, G. L., Thomson, J., \& El -Omar, E. M. (2011). The inflammatory microenvironment in colorectal neoplasia. PLoS One. Jan 7 , 6(1), e15366.

[73] Rajkumar, T., Shirley, S., Raja, U. M., \& Ramakrishnan, S. A. Identification and validation of genes involved in gastric tumorigenesis. Cancer Cell Int., 10, 45. 
[74] Huang, S., Mills, L., Mian, B., Tellez, C., Mc Carty, M., Yang, X. D., Gudas, J. M., \& Bar-Eli, M. (2002). Fully humanized neutralizing antibodies to interleukin-8 (ABXIL8) inhibit angiogenesis, tumor growth, and metastasis of human melanoma. Am J Pathol., 161(1), 125-34.

[75] Mc Conkey, D. J., \& Bar-Eli, M. (2003). Fully human anti-interleukin 8 antibody inhibits tumor growth in orthotopic bladder cancer xenografts via down-regulation of matrix metalloproteases and nuclear factor-kappaB. Clin Cancer Res., 9(8), 3167-75.

[76] Jiang, Z., Xu, Y., \& Cai, S. (2010). CXCL10 expression and prognostic significance in stage II and III colorectal cancer. Mol Biol Rep., 37(6), 3029-36.

[77] Utoguchi, N. H., Makimoto, Y., Wakai, Y., Tsutsumi, S., Nakagawa, , \& Mayumi, T. (1996). Effect of tumour cell-conditioned medium on endothelial macromolecular permeability and its correlation with collagen. British Journal of Cancer, 73, 24-28.

[78] Cao, Z., Xu, X., Luo, X., Li, L., Huang, B., Li, X., Tao, D., Hu, J., \& Gong, J. J. (2011). Huazhong Role of RANTES and its receptor in gastric cancer metastasis. Univ Sci Technolog Med Sci. Epub Jun, 31(3), 342-7.

[79] The high level of RANTES in the ectopic milieu recruits macrophages and induces their tolerance in progression of endometriosis. (2010). J Mol Endocrinol, 45, 291-299.

[80] Lu, H., Ouyang, W., \& Huang, C. (2006). Inflammation, a key event in cancer development. Mol Cancer Res, 4, 221-233.

[81] Yan, B., Wang, H., Rabbani, Z. N., Zhao, Y., Li, W., Yuan, Y., Li, F., Dewhirst, M. W., \& Li, C. Y. (2006). Tumor necrosis factor-alpha is a potent endogenous mutagen that promotes cellular transformation. Cancer Res, 66, 11565-11570.

[82] Kumar et.al. (2010). Robbins and Cortan structural and functional pathology. ed. the eighth edition. Elsevier sounders Barcelona Spain., 19-20.

[83] Hikman, Elizabeth Oldham. (2004). Intrinsic oxidative stress in cancer cells a biological basis for therapeutic selectivity" Cancer". Cancer chemother pharmacol, 53, 209-19.

[84] Cerutti, P. (1985). Pro-oxidant states and tumor promotion. Science, 227, 375-80.

[85] Migliori, L., et al. (1991). Genetic and environmental factors in cancer an neurodegenerative disease. Mut Res, 202(512), 135-153.

[86] Kouchakgjian, M., et al. (1991). MR structural studies of the ionizing radiation adduct 7-hydro-8oxodeoxyguanosine (8-oxo-7H-dG) opposites deoxyadenisine in a D A duplex 8-oxo-7H-7dG(syn)-dA(anti)aligment a lesion site. Biochem, 30, 1403.

[87] Elejalde Guerra, J. I. (2001). Oxidative Stress, diseases and antioxidants treatments. An Med Int (Madrid-Spain), 18, 326-335.

[88] Aherne, S. A., \& y O’Brien, N. M. (2002). Dietary flavonols: chemistry, food content, and metabolism. Nutrition, 18, 75-81. 
[89] Jovanovic, S. V., Steenken, S., Simic, M. G. y., \& Hara, Y. (1998). Antioxidant properties of flavonoids: reduction potentials and electron transfer reactions of flavonoid radicals. En: Rice Evans C, Parker L (eds.): Flavonoids in health and disease. Marcel Dekker, Nueva York, 137-161.

[90] Letan, A. (1966). The relation of structure to antioxidant activity of quercitin and some of its derivates. J Food Sci, 31, 518-523.

[91] Stahl, W., Ale-Agha, N. Y., \& Polidori, M. C. (2002). Non-antioxidant properties of carotenoids. Biol Chem, 383, 553-558.

[92] Stacvric, B. (1994). Quercitin in our diet: From potent mutagen to probable anticarcinogen. Clinical Biochemistry, 27, 245-248.

[93] Da Silva, J., Herrmann, S. M., Peres, W., Possa, Marroni. N., Gonzalez Gallego, J. Y., \& Erdtmann, B. (2002). Evaluation of the genotoxic effect of rutin and quercetin by comet assay and micronucleus test. Food Chem Toxicol, 40, 941-947. 